\title{
3D Lung Tumor Motion Model Extraction from 2D Projection Images of Mega-voltage Cone Beam CT via Optimal Graph Search
}

\author{
Mingqing Chen ${ }^{1,2}$, Junjie Bai ${ }^{1}$, Yefeng Zheng ${ }^{3}$, and R. Alfredo C. Siochi ${ }^{2}$ \\ 1 Department of Electrical and Computer Engineering \\ 2 Department of Radiation Oncology, University of Iowa, Iowa City, IA, USA \\ 3 Image Analytics and Informatics, Siemens Corporate Research, Princeton, NJ, USA \\ mingqing-chen@uiowa.com
}

\begin{abstract}
In this paper, we propose a novel method to convert segmentation of objects with quasi-periodic motion in $2 \mathrm{D}$ rotational cone beam projection images into an optimal 3D multiple interrelated surface detection problem, which can be solved by a graph search framework. The method is tested on lung tumor segmentation in projection images of mega-voltage cone beam CT (MVCBCT). A 4D directed graph is constructed based on an initialized tumor mesh model, where the cost value for this graph is computed from the point location of a silhouette outline of projected tumor mesh in 2D projection images. The method was first evaluated on four different sized phantom inserts (all above $1.9 \mathrm{~cm}$ in diameter) with a predefined motion of $3.0 \mathrm{~cm}$ to mimic the imaging of lung tumors. A dice coefficient of $0.87 \pm 0.03$ and a centroid error of $1.94 \pm 1.31 \mathrm{~mm}$ were obtained. Results based on 12 MVCBCT scans from 3 patients obtained $0.91 \pm 0.03$ for dice coefficient and $1.83 \pm 1.31 \mathrm{~mm}$ for centroid error, compared with a difference between two sets of independent manual contours of $0.89 \pm 0.03$ and $1.61 \pm 1.19 \mathrm{~mm}$, respectively.
\end{abstract}

\section{Introduction}

The recent advances of mega-voltage cone beam computed tomography (MVCBCT) [1] have enabled the use of linear accelerator (linac) treatment beams for cone beam imaging. This development provides an imaging solution of patient localization to verify the positioning and anatomy information prior to treatment delivery. The $3 \mathrm{D}$ volumetric image has potential to improve the accuracy for correcting target misalignments and verifying the treatment plan [2.

However, when the system is used for non-small cell lung cancer (NSCLC) imaging, the existence of respiratory motion during image acquisition causes blurring and streaking artifacts. This motion-blurred 3D volumetric image alone cannot provide much information about tumor size and the motion model, which can change significantly over the full course of a fractionated treatment. It is highly desirable to derive the tumor motion information during the localization scan prior to treatment delivery.

One promising solution is to use cone beam projections to detect tumor motion, since they have high temporal resolution. Previous methods include (1) 


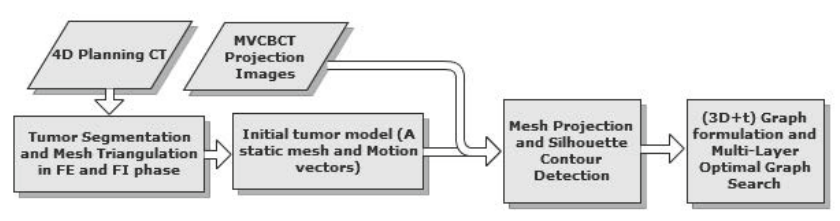

Fig. 1. Flowchart of the proposed approach

monitoring tumor change by projecting a volume of interest for visualization [3], (2) extracting the $2 \mathrm{D} / 3 \mathrm{D}$ position of a projected implanted marker [4] or diaphragm edge [5], which can also be used as respiratory signal for gated reconstruction, and (3) registering from 3D image space to projection space for inter-phase motion compensated reconstruction [6. Direct tumor tracking or detection in 2D images is mainly focused on fluoroscopy [7. However, few studies have addressed direct tumor detection in MVCBCT projection images, which suffer from relatively poor contrast and the interfering anatomies.

In this study, we present a novel method based on an optimal graph search framework [8] to extract a $(3 \mathrm{D}+\mathrm{t})$ tumor motion model from $2 \mathrm{D}$ projection images. Two major advantages make the method robust in the low-contrast images: (1) The 3D tumor segmentation is based on all the $2 \mathrm{D}$ projection images that belong to the corresponding respiratory phase. The detection inaccuracies induced by low contrast and interference of one projection image can thus be reduced. (2) Compared with other 2D-to-3D object shape recovery methods, such as free form deformation [9], B-splines surface model [10] and triangulated mesh pulling [11], our approach incorporates both motion and shape constraints in the segmentation process and obtains a global optimal solution.

\section{Method}

\subsection{General Framework}

The main steps of the proposed approach are illustrated in Fig. 1 with the intermediate results shown in Fig. 2. In preparation for the algorithm, the projection images are sorted into several respiratory phase bins according to the $3 \mathrm{D}$ anatomical positions of the ipsi-lateral hemi-diaphragm apex (IHDA), which is automatically extracted from projection images based on the dynamic Hough transform [12. The proposed algorithm starts with an initial 3D static lung tumor mesh model, which reflects the approximate topological structure information of the targeted tumor surface. The initial tumor mesh is projected onto each $2 \mathrm{D}$ projection image. The new location of mesh points for all the respiratory bins are determined simultaneously using a multi-surface optimal graph search method [8], which requires computation of the silhouette outline for each projected mesh at first. 


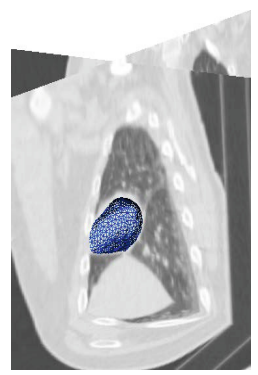

(a)

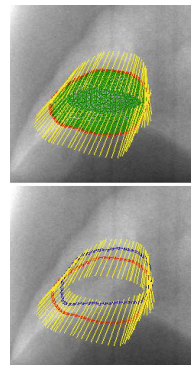

(b)

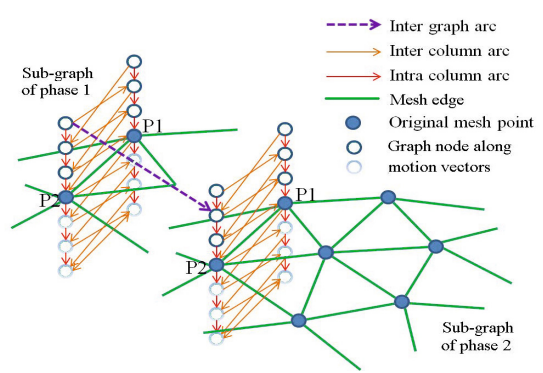

(c)

Fig. 2. (a) Full exhale phase of 4D diagnostic CT volume overlaid with tumor mesh (blue); (b) Projected initial static mesh (green), its silhouette contour (red), motion direction (yellow) and the silhouette contour after the graph search computation (blue); (c) A simple illustration of the $4 \mathrm{D}$ graph construction

\subsection{Model Initialization}

The initial static model is the average of the meshes segmented from the full exhale (FE) and full inhale (FI) phases of the 4DCT. For each mesh point, a range of motion is determined using the equation $\left[P_{m}+\alpha\left(P_{f e}-P_{m}\right)\right]$ and $\left[P_{m}+\alpha\left(P_{f i}-P_{m}\right)\right]$, where $P_{f e}$ and $P_{f i}$ is the corresponding positions in the FE and FI phase, respectively, and $P_{m}$ is the mean position. $\alpha$ is used to control the allowed range, which is typically set between 1.5 and 2 . The two meshes are initialized with the same spherical mesh and manually adjusted according to the tumor boundary in the $3 \mathrm{D}$ volume using our in-house platform. Thus the correspondence of mesh points is inherently established.

\subsection{Silhouette Contour Extraction}

The initial mesh, along with pre-defined motion vectors, is projected onto each $2 \mathrm{D}$ projection image. In order to move the mesh towards tumor boundary locations in the projection image, the silhouette outline is extracted from each projected mesh by using an efficient algorithm [11. An example of the detected silhouette outline is shown in Fig. 2.

\subsection{Multiple Surface Detection via Optimal Graph Search}

A key innovation of the proposed method is converting the segmentation of objects with quasi-periodic motion in $2 \mathrm{D}$ rotational cone beam projection images into a 3D multiple interrelated surface detection problem, which can be solved by a graph search framework [8]. The details are presented as follows. A 4D $(3 \mathrm{D}+\mathrm{t})$ directed graph $G=(V, E)$ is constructed based on the initial tumor mesh. The graph contains $T$ (number of phase bins) subgraphs, where each subgraph corresponds to the tumor surface in one respiratory phase bin. Each subgraph contains $N \times M$ nodes, where $N$ and $M$ are the number of points of the static tumor mesh and the number of sampled points along the pre-defined motion vector. Each combination of $[n, m, t]$ is one unique spatial and temporal location, which represents the $m$ th sampled point in the column defined by 
mesh point $n$ in phase $t$. The segmented tumor surfaces are defined by function $\mathcal{N}:(n, t) \rightarrow \mathcal{N}(n, t)$, where $n \in \mathbf{n}=\{0, \ldots, N-1\}, t \in \mathbf{t}=\{0, \ldots, T-1\}$, and $\mathcal{N}(n, t) \in \mathbf{m}=\{0, \ldots, M-1\}$.

A cost value is computed for each node $[n, m, t]$, denoted by $c(n, m, t)$, using the following equation:

$$
c(n, m, t)=\sum_{p=0}^{P-1} \delta(p, t) \xi(n, p) w(n, m, p),
$$

where $P$ and $p$ is the total number and the index of projection images, respectively. The function $\delta(p, t)=1$ when the $p$ th projection image belongs to the $t$ th bin, otherwise it equals zero. The function $\xi(n, p)=1$ when the $n$th point is included in the silhouette contour of the $p$ th projection image, otherwise it equals zero. $w(n, m, p)$ is the cost function of the $m$ th sample point in the $n$th column in the $p$ th projection image, which is defined as:

$$
w(n, m, p)=-\ddot{P}_{p}(\operatorname{normal}(n)) \cdot \operatorname{grad}\left(\ddot{P}_{p}(\mathbf{P}(n, m))\right),
$$

where $\ddot{P}_{p}$ is the 3 D-to- 2 D projection operation of a vector or point in the $p$ th projection image. $\mathbf{P}(n, m)$ is the location of the $m$ th sample point along the predefined motion vector of the $n$th mesh point. The operation normal $(n)$ gives the normal direction of the $n$th point of the static mesh, while the operation grad computes the image gradient of a given 2D location. The reason for using the negative dot product between these two vectors is that along the tumor boundary in 2D projection images, the projected normal direction is opposite to the image gradient. Equations (11) and (2) show that the cost for each node in the $4 \mathrm{D}$ graph is determined from all the $2 \mathrm{D}$ projection images that belong to the corresponding respiratory bin.

Three different types of arcs are added to the graph: (1) Intra-column arcs are used to define the graph topology, which connect adjacent nodes that belong to the same column. The arc goes from each node $[n, m, t](m>0)$ to the node below $[n, m-1, t]$. (2) Inter-column arcs are used to connect adjacent columns in the same respiratory bin. The arc goes from each node $[n, m, t]\left(m>\delta_{m}\right)$ to $\left[\operatorname{adj}(n), m-\delta_{m}, t\right]$, where $\operatorname{adj}(n)$ represents adjacent mesh points of $n . \delta_{m}$ is the shape smoothness constraint, which is the maximal allowed difference in $m$ between adjacent columns of one tumor surface. (3) Inter-phase arcs are used to connect the same columns in adjacent respiratory bins. The arc goes from each node $[n, m, t]\left(m>\delta_{t}\right)$ to $\left[n, m-\delta_{t}, t \pm 1\right] . \delta_{t}$ is the inter-phase constraint, which is the maximal allowed difference in $m$ between adjacent bins of the same column. We define that $[n, m, 0]=[n, m, T]$ to form a closed loop of respiratory bins. Fig. 2r illustrates the main idea for graph construction, where a simple case of $T=2, M=N=7, \delta_{m}=\delta_{t}=1$ is shown, where two subgraphs representing the two phases are shown. The forementioned three types of arcs are illustrated. For visualization purposes, only one inter-phase arc is drawn. And only two columns are shown for each subgraph. The subgraph of phase 2 shows all the mesh points, while phase 1 only shows the two points with columns. In our implementation, $N$ 
is typically from 1000 to 4000 , depending on the tumor size. $M$ is 50 , making the step size of the sampled point equal to $1 / 50$ of the allowed range of motion. $T$ is 20, where larger $T$ makes higher temporal resolution, but reduces the number of projections for each phase bin. The optimal solution can be computed by solving a maximal flow problem in the constructed graph [8]. The running time based on those parameters is about 40s on an Intel Core $^{T M}$ I7 laptop with 4 GB RAM.

\section{Experiments}

\subsection{Imaging Data}

Our clinic is equipped with a Siemens Oncor MVCBCT system (Siemens Medical Systems, Oncology Care Systems, Concord, CA) with an electrical portal imaging device (EPID) to acquire 2D projection images. Using the standard protocol [1, the 200 EPID projection images are acquired as the gantry rotates clockwise from $-90^{\circ}$ to $110^{\circ}$ in about 1 minute.

The proposed approach was first verified on an imaging phantom, which has a predefined motion and size to serve as the ground truth. The phantom consists of a block of basswood and six different sized spherical inserts. The basswood frame has a density of about $0.4 \mathrm{~g} / \mathrm{cc}$ to mimic lung tissue, while the six inserts are made of paraffin wax and have $3.81,3.18,2.54,1.91,0.95$, and $0.48 \mathrm{~cm}$ in diameter, respectively. Fig. 3 shows a picture of the phantom, a coronal slice of a diagnostic $\mathrm{CT}$ and an MVCBCT projection image, respectively. The phantom is placed on a cart attached to the Quasar respiratory motion (QRM) phantom (Modus Medical Devices, INC, London, ON, Canada) to simulate respiratory motion. The QRM phantom is programmed to move only in the superior-inferior (SI) direction, with its position as a function of time $t$, defined as:

$$
z(t)=z_{0}+A_{0} \cos ^{4}\left(\pi\left(t+t_{0}\right) / \tau\right)
$$

where the motion amplitude $A_{0}$ is $30 \mathrm{~mm}$, and the period $\tau$ is $4 s$ to represent typical breathing. $z_{0}$ and $t_{0}$ are the DC component of the motion and the starting phase of the phantom motion, which varies among different experiments. The phantom tests were done on two scans, with a dose of 5MU and 10MU, respectively.

The proposed method was also tested on 12 scans from three patients, who have relatively large tumors in the lower lobe of the lung. The patient scans used an imaging dose of $10 \mathrm{MU}$.

\subsection{Results}

Fig. 4] shows the detection result of the largest insert of the phantom (top) and a real patient whose tumor is right above the diaphragm (bottom). The top-right corner shows the detected $3 \mathrm{D}$ mesh in the corresponding respiratory bin. The evaluation is based on $2 \mathrm{D}$ contours in projection space. For the phantom images, the detected contour of the inserts is compared with the contour computed from the predefined size and the motion, which is considered ground truth. It can be seen that the insert can be detected robustly in the presence of the interfering superimposed objects, 

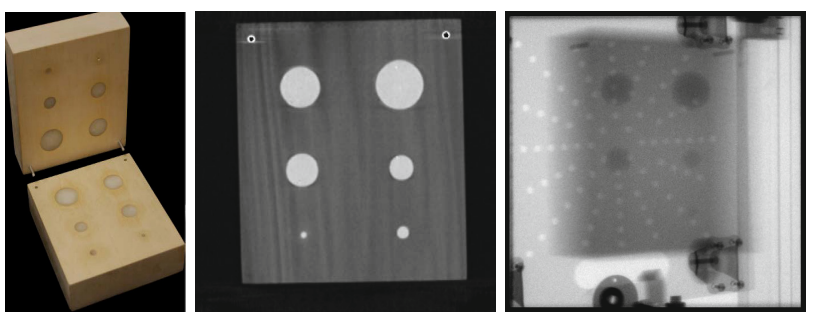

Fig. 3. Left: the imaging phantom; Middle: a coronal slice of the FE phase of the $4 \mathrm{D}$ diagnostic CT; Right: one projection image of MVCBCT with a dose of $10 \mathrm{MU}$
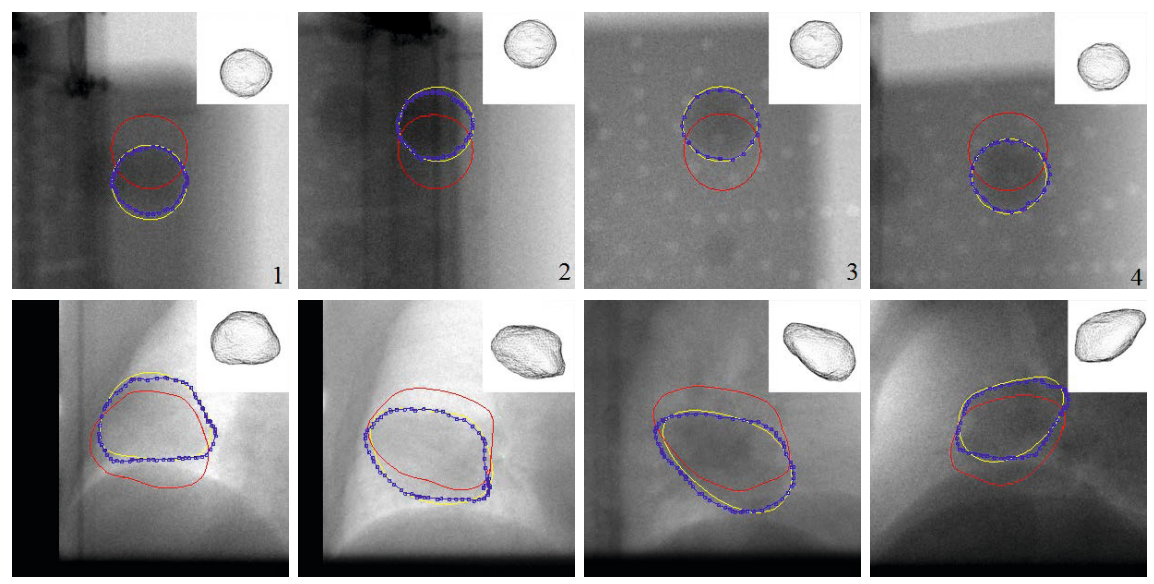

Fig. 4. Detection result on Top row: the imaging phantom; Bottom row: a patient with tumor above the diaphragm. Red: silhouette outline of initial mesh; blue: detected tumor contour (deformed silhouette outline); yellow: contour of ground truth for phantom images, manually annotated contour for patient images. The detected 3D tumor mesh of the corresponding respiratory bin is displayed on the top-right corner.

such as interfering spheres (Fig.41), the QRM motion phantom (Fig.442) and the

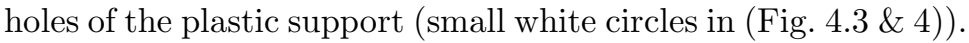

The evaluation is based on the four largest inserts, since there is no strong boundary information of the two smaller ones in the projection images (Fig. (3). Two metrics were employed to validate the detection result: the $2 \mathrm{D}$ dice coefficient and the difference of centroid positions along the SI direction. Fig. [5 shows the mean and standard deviation values of those metrics over 200 projection images. The dice coefficient decreases slightly when the tumor size goes down, while this phenomenon does not occur in the centroid error. An imaging dose of $10 \mathrm{MU}$ obtains better accuracy for centroid and a slight improvement in the dice coefficient. For patient images, the tumor was independently contoured by two clinical experts. The averaged contour was computed to compare with the detection result. The difference between the two manual contours is also quantified. Fig. [6 

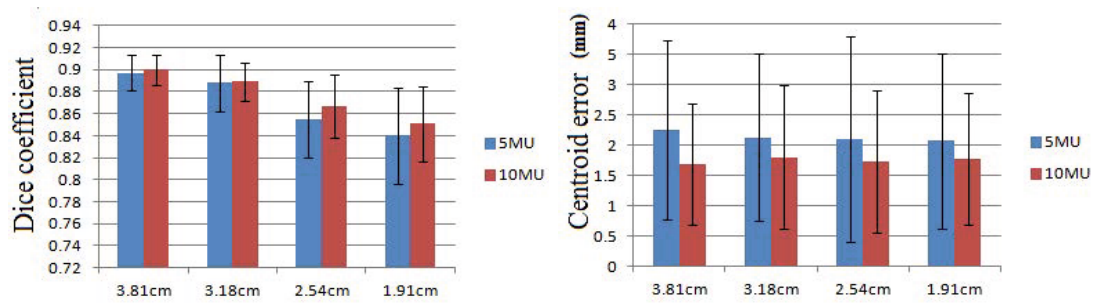

Fig. 5. Mean and standard deviation of Left: dice coefficient between detected contour and ground truth; Right: centroid difference between detected contour and ground truth over 200 projection images of four spherical inserts
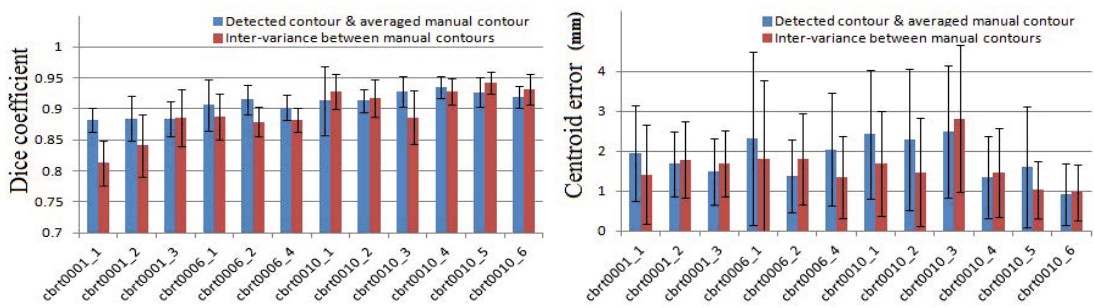

Fig. 6. Mean and standard deviation of Left: dice coefficient; Right: centroid difference over 200 projection images of 12 patient MVCBCT scans

shows the dice coefficient and centroid difference over 12 MVCBCT scans from 3 patients, where the overall dice coefficient of the proposed method is even better than variations between manual contours. This result is expected, since the segmentation of the $3 \mathrm{D}$ shape is based on multiple $2 \mathrm{D}$ views, while the manual contour could be confounded by overlapping tissues in one single image. For both phantom and patient studies, the quantified centroid error is clinically acceptable, since a setup error of $5 \mathrm{~mm}$ is typically added to the gross tumor volume (GTV) for treatment delivery.

\section{Discussion and Conclusion}

In this work, we proposed a novel method to extract the $3 \mathrm{D}$ tumor motion model from 2D projection images of an MVCBCT system. Experiments based on phantom images show the robustness to detect tumors with diameters larger than $1.9 \mathrm{~cm}$. For patient images, the method can even be used for guidance to assist the clinician for a better visualization of tumor boundaries. The segmentation framework is based on a simple scaling model between the FE and FI phases for each mesh point. Though in reality the motion trajectory is more like an elongated ellipse, the linear scaling model is a good estimation and it has enabled us to derive accurate detection result. It is potentially helpful to use a more complicated model established from multiple phases of 4D CT. However, more computation and complexity will be added to the current framework. 
In the patient studies, the shape of the segmentation changes somehow over the respiratory cycle. The tumor mass for those patients are relatively large and they are likely to be deformed in a non-rigid way by the pressure of surrounding tissues and the diaphragm. The shape and motion constraint in the $4 \mathrm{D}$ graph can further be adjusted to control the degree of shape change. The method can be easily applied to other organs with quasi-periodic motion, such as the cardiac chambers or lungs. It can also be extended to other cone beam systems, such as the $\mathrm{C}$-arm systems. In the future, more patient data will be tested with various tumor size and shape, imaging dose and breathing pattern.

\section{References}

1. Morin, O., Gillis, A., Chen, J., Aubin, M., Bucci, M., Roach III, M., Pouliot, J.: Megavoltage cone-beam CT: system description and clinical applications. Medical Dosimetry 31(1), 51-61 (2006)

2. Jaffray, D., Siewerdsen, J., Wong, J., Martinez, A.: Flat-panel cone-beam computed tomography for image-guided radiation therapy. International Journal of Radiation Oncology Biology Physics 53(5), 1337-1349 (2002)

3. Reitz, B., Gayou, O., Parda, D., Miften, M.: Monitoring tumor motion with on-line mega-voltage cone-beam computed tomography imaging in a cine mode. Physics in Medicine and Biology 53, 823-836 (2008)

4. Li, T., Xing, L., Munro, P., McGuinness, C., Chao, M., Yang, Y., Loo, B., Koong, A.: Four-dimensional cone-beam computed tomography using an on-board imager. Medical Physics 33, 3825-3833 (2006)

5. Siochi, R.: Deriving motion from megavoltage localization cone beam computed tomography scans. Physics in Medicine and Biology 54, 4195-4212 (2009)

6. Li, T., Koong, A., Xing, L.: Enhanced 4D cone-beam CT with inter-phase motion model. Medical Physics 34, 3688-3695 (2007)

7. Shimizu, S., Shirato, H., Ogura, S., Akita-Dosaka, H., Kitamura, K., Nishioka, T., Kagei, K., Nishimura, M., Miyasaka, K.: Detection of lung tumor movement in realtime tumor-tracking radiotherapy. International Journal of Radiation Oncology Biology Physics 51(2), 304-310 (2001)

8. Li, K., Millington, S., Wu, X., Chen, D.Z., Sonka, M.: Simultaneous Segmentation of Multiple Closed Surfaces Using Optimal Graph Searching. In: Christensen, G.E., Sonka, M. (eds.) IPMI 2005. LNCS, vol. 3565, pp. 406-417. Springer, Heidelberg (2005)

9. Lotjonen, J., Magnin, I., Nenonen, J., Katila, T.: Reconstruction of 3-D geometry using 2-D profiles and a geometric prior model. IEEE Trans. Medical Imaging 18(10), 992-1002 (1999)

10. Moriyama, M., Sato, Y., Naito, H., Hanayama, M., Ueguchi, T., Harada, T., Yoshimoto, F., Tamura, S.: Reconstruction of time-varying 3D left ventricular shape from multiview x-ray cineangiocardiograms. IEEE Trans. Medical Imaging, 773-785 (2002)

11. Chen, M., Zheng, Y., Mueller, K., Rohkohl, C., Lauritsch, G., Boese, J., Funka-Lea, G., Hornegger, J., Comaniciu, D.: Automatic Extraction of 3D Dynamic Left Ventricle Model from 2D Rotational Angiocardiogram. In: Fichtinger, G., Martel, A., Peters, T. (eds.) MICCAI 2011, Part III. LNCS, vol. 6893, pp. 471-478. Springer, Heidelberg (2011)

12. Chen, M., Siochi, R.: Diaphragm motion quantification in megavoltage cone-beam CT projection images. Medical Physics 37, 2312-2320 (2010) 